\title{
EVIDENCE OF ANGULAR MOMENTUM LOSS IN THE ECLIPSING BINARY VW CEPHEI
}

\author{
M. J. DEVITA AND D. H. BRADSTREET \\ Eastern College, St. Davids, PA \\ mdevita@eastern.edu \\ E. F. GUINAN \\ Villanova University guinan@ucis.vill.edu \\ AND \\ Z. GLOWNIA \\ Palomar College glownia@minaka.sdsu.edu
}

VW Cep is one of the brightest and longest observed short-period ( $\mathrm{P}=6.67$ hours) W UMa type binaries. It consists of a G5V and $\mathrm{K} 0 \mathrm{~V}$ components in contact with their Roche surfaces. We investigated complex period changes based upon eclipse timings from the past 70 years. In addition to the well-known 30 year light time effect due to the presence of a third star in the system, we find evidence for a long term decrease in the orbital period of $\mathrm{dP} / \mathrm{dt}=-0.02 \mathrm{sec} / \mathrm{yr}$. This decrease in period could arise from angular momentum loss from the binary or mass exchange between components. From these timings we have refined the properties of the tertiary component and redetermined its mass and orbital parameters. After correcting the O-C's for the third body and the steady decrease in Keplerian period, we uncovered small systematic deviations in the residual $\mathrm{O}-\mathrm{C}$ 's. We are studying these second order period variations, possibly arising from mass loss in winds and/or mass flow between components, to determine if they correlate with known cycles of spot and surface activity. 